\title{
OSCILLATION OF SECOND ORDER LINEAR ORDINARY DIFFERENTIAL EQUATIONS WITH ALTERNATING COEFFICIENTS
}

\author{
Ch,G. Philos
}

\begin{abstract}
A new result is obtained for the oscillation of second order linear ordinary differential equations with alternating coefficients. This oscillation result extends a recent oscillation criterion due to Kamenev [Mat. Zametki 23 (1978), 249-251].
\end{abstract}

Consider the second order linear ordinary differential equation

$$
x^{\prime \prime}(t)+a(t) x(t)=0, \quad t \geq t_{0},
$$

where $a$ is a continuous real-valued function on the interval $\left[t_{0}, \infty\right)$ without any restriction on its sign. A solution of (E) is said to be oscizlatory if the set of its zeros is unbounded above, and otherwise it is said to be nonoscilzatory. Equation (E) is called oscillatory if all its solutions are oscillatory.

For the oscillation of the equation (E) the following integral criterion due to Wintner [4] is well-known. If

$$
\lim _{t \rightarrow \infty} \frac{I}{t} \int_{t_{0}}^{t}(t-s) a(s) d s=\infty
$$

then (E) is oscizlatory. Hartman [1] has shown that the limit cannot be replaced by the upper limit in the above condition. Recently, Kamenev [2]

Received 10 December 1982. 
has proved a new integral criterion for the oscillation of the differential equation ( $E$ ), based on the use of the $n$th primitive of the coefficient $a$, which has the result of Wintner as a particular case. More precisely, Kamenev has established that $(E)$ is oscillatory if, for some integer $n \geq 3$,

$\left(C_{0}\right)$

$$
\limsup _{t \rightarrow \infty} \frac{1}{t^{n-1}} \int_{t_{0}}^{t}(t-s)^{n-1} a(s) d s=\infty
$$

Kamenev's criterion has been extended in various directions by Yeh [5], [6] and Philos [3]. The purpose of this paper is to extend the result of Kamenev by multiplying the coefficient $a$ in condition ( $\mathrm{C}_{0}$ ) by a function which belongs to an appropriate function class depending on the integer $n$. More precisely, our main result is the following theorem.

THEOREM. Let $n$ be an integer with $n \geq 3$ and $\rho$ be a positive continuously differentiable function on the interval $\left[t_{0}, \infty\right)$ such that

$$
\lim _{t \rightarrow \infty} \sup \frac{1}{t^{n-1}} \int_{t_{0}}^{t} \frac{(t-s)^{n-3}}{\rho(s)}\left[(n-1) \rho(s)-(t-s) \rho^{\prime}(s)\right]^{2} d s<\infty .
$$

Equation (E) is oscillatory if

$$
\underset{t \rightarrow \infty}{\lim \sup } \frac{1}{t^{n-1}} \int_{t_{0}}^{t}(t-s)^{n-1} \rho(s) a(s) d s=\infty \text {. }
$$

Proof. Let $x$ be a nonoscillatory solution of the differential equation $(E)$ and let $T>\max \left\{t_{0}, 0\right\}$ be such that $x(t) \neq 0$ for all $t \geq T$. As in [2], we set $w=x^{\prime} / x$ on $[T, \infty)$ and we obtain

$$
a(t)=-w^{\prime}(t)-w^{2}(t) \text { for every } t \geq T .
$$

Then for $t \geq T$ we have

$$
\begin{aligned}
\int_{T}^{t}(t-s)^{n-1} \rho(s) a(s) d s \\
\quad=-\int_{T}^{t}(t-s)^{n-1} \rho(s) \omega^{\prime}(s) d s-\int_{T}^{t}(t-s)^{n-1} \rho(s) \omega^{2}(s) d s
\end{aligned}
$$




$$
\begin{aligned}
& =(t-T)^{n-1} \rho(T) w(T)+\frac{1}{4} \int_{T}^{t} \frac{(t-s)^{n-3}}{\rho(s)}\left[(n-1) \rho(s)-(t-s) \rho^{\prime}(s)\right]^{2} d s \\
& -\int_{T}^{t}\left\{(t-s)^{(n-1) / 2} \sqrt{\rho(s)} w(s)+\frac{(t-s)^{(n-3) / 2}\left[(n-1) \rho(s)-(t-s) \rho^{\prime}(s)\right]}{2 \sqrt{\rho(s)}}\right\}^{2} d s \\
& \leq(t-T)^{n-1} \rho(T) w(T)+\frac{1}{4} \int_{t_{0}}^{t} \frac{(t-s)^{n-3}}{\rho(s)}\left[(n-1) \rho(s)-(t-s) \rho^{\prime}(s)\right]^{2} d s \text {. }
\end{aligned}
$$

On the other hand, for every $t \geq T$, we get

$$
\begin{aligned}
\int_{t_{0}}^{t}(t-s)^{n-1} \rho(s) a(s) d s-\int_{T}^{t}(t-s)^{n-1} \rho(s) a(s) d s \\
\quad=\int_{t_{0}}^{T}(t-s)^{n-1} \rho(s) a(s) d s \\
\leq \int_{t_{0}}^{T}(t-s)^{n-1} \rho(s)|a(s)| d s \leq\left(t-t_{0}\right)^{n-1} \int_{t_{0}}^{T} \rho(s)|a(s)| d s .
\end{aligned}
$$

Thus

$$
\begin{aligned}
& \frac{1}{t^{n-1}} \int_{t_{0}}^{t}(t-s)^{n-1} \rho(s) a(s) d s \\
& \leq\left(1-\frac{T}{t}\right)^{n-1} \rho(T) \omega(T)+\left(1-\frac{t_{0}}{t}\right)^{n-1} \int_{t_{0}}^{T} \rho(s)|a(s)|_{d s} \\
& \quad+\frac{1}{t^{n-1}} \int_{t_{0}}^{t} \frac{(t-s)^{n-3}}{\rho(s)}\left[(n-1) \rho(s)-(t-s) \rho^{\prime}(s)\right]^{2} d s
\end{aligned}
$$

for all $t \geq T$. This gives

$$
\begin{array}{r}
\underset{t \rightarrow \infty}{\lim \sup } \frac{1}{t^{n-1}} \int_{t_{0}}^{t}(t-s)^{n-1} \rho(s) a(s) d s \leq \rho(T) w(T)+\int_{t_{0}}^{T} \rho(s)|a(s)| d s \\
\quad+\quad \lim _{t \rightarrow \infty} \sup \frac{1}{t^{n-1}} \int_{t_{0}}^{t} \frac{(t-s)^{n-3}}{\rho(s)}\left[(n-1) \rho(s)-(t-s) \rho^{\prime}(s)\right]^{2} d s,
\end{array}
$$

which contradicts conditions $(H)$ and $(C)$.

REMARK 1. By setting $\rho(t)=1, t \geq t_{0}$, our theorem leads to 
Kamenev's criterion.

Now we remark that $\rho$ satisfies $(H)$ for any integer $n \geq 3$ if:

$\left(\mathrm{H}^{\prime}\right)$

$$
\left\{\begin{array}{c}
\lim \inf _{t \rightarrow \infty} \rho^{\prime}(t)>-\infty, \\
\lim _{t \rightarrow \infty} \sup \frac{1}{t^{2}} \int_{t_{0}}^{t} \rho(s) d s<\infty \text { and } \int^{\infty} \frac{\left[\rho^{\prime}(t)\right]^{2}}{\rho(t)} d t<\infty .
\end{array}\right.
$$

A special case where $\rho$ is subject to $\left(\mathrm{H}^{\prime}\right)$ is that $\rho(t)=t^{\alpha}$, $t \geq t_{0}>0$ for $\alpha \in[0,1)$. So we have the following corollary.

COROLLARY 1. Let $t_{0}>0$. Equation (E) is oscizzatory if there exists $a \quad \alpha \in[0,1)$ such that

$$
\underset{t \rightarrow \infty}{\lim \sup } \frac{1}{t^{n-1}} \int_{t_{0}}^{t}(t-s)^{n-1} \alpha_{a}^{\alpha}(s) d s=\infty \text { for some integer } n \geq 3 \text {. }
$$

Also, when $\rho(t)=\log t$ for $t \geq t_{0}>1$, condition (H') is satisfied by itself. Hence we obtain Corollary 2 below.

COROLLARY 2. Let $t_{0}>1$. Equation (E) is oscizlatory if $\underset{t \rightarrow \infty}{\lim \sup } \frac{1}{t^{n-1}} \int_{t_{0}}^{t}(t-s)^{n-1} \log s \cdot a(s) d s=\infty$ for some integer $n \geq 3_{-}$.

Now let us consider the more general differential equation with a damped term

$$
x^{\prime \prime}(t)+q(t) x^{\prime}(t)+a(t) x(t)=0, t \geq t_{0},
$$

where $q$ is a continuous real-valued function on the interval $\left[t_{0}, \infty\right]$ without any restriction on its sign.

Let $n$ be an integer with $n \geq 3$ and $\rho$ be a positive continuously differentiable function on $\left[t_{0}, \infty\right)$. We consider a nonoscillatory solution $x$ of $\left(E^{*}\right)$ with $x(t) \neq 0, t \geq T$, for some $T>\max \left\{t_{0}, 0\right\}$ and we set $w=x^{\prime} / x$ on $[T, \infty)$. Then $a=-w^{\prime}-q w-w^{2}$ on $[T, \infty)$ and hence, for every $t \geq T$, we obtain 


$$
\begin{aligned}
& \int_{T}^{t}(t-s)^{n-1} \rho(s) a(s) d s \\
& =-\int_{T}^{t}(t-s)^{n-1} \rho(s) w^{\prime}(s) d s-\int_{T}^{t}(t-s)^{n-1} \rho(s) q(s) w(s) d s \\
& =(t-T)^{n-1} \rho(T) w(T)+\frac{1}{4} \int_{T}^{t} \frac{(t-s)^{n-3}}{\rho(s)}\left\{(n-1) \rho(s)+(t-s)\left[\rho(s) q(s)-\rho^{\prime}(s)\right]\right\}^{2} d s \\
& -\int_{T}^{t}\left\{(t-s)^{n-1} \sqrt{\rho(s)} w(s)+\frac{(t-s)^{(n-3) / 2}\left\{(n-1) \rho(s)+(t-s)\left[\rho(s) q(s)-\rho^{\prime}(s)\right]\right\}}{2 \sqrt{\rho(s)}}\right\}_{T}^{2}(t-s)^{n-1} \rho(s) w^{2}(s) d s \\
& \leq(t-T)^{n-1} \rho(T) w(T)+\frac{1}{4} \int_{t_{0}}^{t} \frac{(t-s)^{n-3}}{\rho(s)}\left\{(n-1) \rho(s)+(t-s)\left[\rho(s) q(s)-\rho^{\prime}(s)\right]\right\}^{2} d s .
\end{aligned}
$$

Thus we derive the following generalization of our theorem.

Let $n$ be an integer with $n \geq 3$ and $\rho$ be a positive continuously differentiable function on the interval $\left[t_{0}, \infty\right)$. Suppose that

$$
\left(H_{1}\right) \quad \underset{t \rightarrow \infty}{\lim \sup } \frac{1}{t^{n-1}} \int_{t_{0}}^{i} \frac{(t-s)^{n-3}}{\rho(s)}\left\{(n-1) \rho(s)+(t-s)\left[\rho(s) q(s)-\rho^{\prime}(s)\right]\right\}^{2} d s<\infty
$$

Equation ( $\left.E^{*}\right)$ is oscillatory if (C) holds.

We remark that $\left(\mathrm{H}_{1}\right)$ holds for any integer $n \geq 3$ if the function $\rho$ satisfies

$\left(\mathrm{H}_{1}^{\prime}\right)\left\{\begin{array}{l}\lim \inf _{t \rightarrow \infty}\left[\rho^{\prime}(t)-\rho(t) q(t)\right]>-\infty, \\ \lim _{t \rightarrow \infty} \sup _{t} \frac{1}{t^{2}} \int_{t_{0}}^{t} \rho(s) d s<\infty \text { and } \int^{\infty} \frac{\left[\rho^{\prime}(t)-\rho(t) q(t)\right]^{2}}{\rho(t)} d t<\infty .\end{array}\right.$

Also we note that for $\rho(t)=1, t \geq t_{0}$, condition $\left(H_{1}\right)$ becomes

$\left(\mathrm{H}_{1}^{\prime \prime}\right) \quad \lim \sup _{t \rightarrow \infty} \frac{1}{t^{n-1}} \int_{t_{0}}^{t}(t-s)^{n-3}[n-1+(t-s) q(s)]^{2} d s<\infty$.

So we obtain the following result due to Yeh [6]. 
Equation ( $\left.\mathrm{E}^{*}\right)$ is oscillatory if, for some integer $n \geq 3,\left(\mathrm{C}_{0}\right)$ and $\left(\mathrm{H}_{1}^{\prime \prime}\right)$ hold.

REMARK 2. It is easy to see that (cf. [3], [5] and [6]) the results obtained for the differential equation (E) or for the more general equation $\left(E^{*}\right)$ hold also for the case of the not necessarily linear differential equation

$$
x^{\prime \prime}(t)+a(t) f[x(t)]=0, \quad t \geq t_{0},
$$

or for the case of the equation

$$
x^{\prime \prime}(t)+q(t) x^{\prime}(t)+a(t) f[x(t)]=0, \quad t \geq t_{0},
$$

respectively, where $f$ is a continuous function on the real line $R$ which is differentiable on $R-\{0\}$ and such that, for some constant $k>0$,

$$
y f(y)>0 \text { and } f^{\prime}(y) \geq k \text { for } y \neq 0 \text {. }
$$

REMARK 3. With the use of some well-known transformations the results of this paper can be extended for more general differential equations involving the term $\left(r x^{\prime}\right)^{\prime}$ in place of the second derivative of the unknown function $x$, where $r$ is a positive continuous function on the interval $\left[t_{0}, \infty\right)$.

\section{References}

[1] Philip Hartman, "On non-oscillatory linear differential equations of second order", Amer. J. Math. 74 (1952), 389-400.

[2] И.В. Каменев [1.v. Kamenev], "0б одном интегральном признаке калеблемости линейных дифереицальндх уравнений второго иоряпка" [An integral criterion for oscillation of linear differential equations of second order", Mat. Zametki 23 (1978), 249-251.

[3] Ch.G. Philos, "On a Kamenev's integral criterion for oscillation of linear differential equations of second order", Utilitas Math. (to appear).

[4] Aurel Wintner, "A criterion of oscillatory stability", Quart. Appl. Math. 7 (1949), 115-117. 
[5] Cheh-Chih Yeh, "An oscillation criterion for second order nonlinear differential equations with functional arguments", J. Math. Anal. Appl. 76 (1980), 72-76.

[6] Cheh-Chih Yeh, "Oscillation theorems for nonlinear second order differential equations with damped term", Proc. Amer. Math. Soc. $84(1982), 397-402$.

Department of Mathematics, University of loannina, loannina, Greece. 